\title{
WEIGHTED POLYNOMIALS ON FINITE AND INFINITE INTERVALS: A UNIFIED APPROACH
}

\author{
BY H. N. MHASKAR AND E. B. SAFF ${ }^{1}$
}

1. Introduction. As described in the survey article [6], the study of "incomplete polynomials", as introduced by G. G. Lorentz [4] in 1976, leads to results on the asymptotic properties of polynomials orthogonal on an infinite interval (cf. [5]) and to theorems on the convergence of "ray sequences" of Padé approximants for Stieltjes functions. Here we present a generalization of the theory for incomplete polynomials which unifies many of the previous results. The essential question which serves as the starting point for the investigation is the following: ${ }^{1}$

Suppose $w(x)$ is a nonnegative weight function continuous on its support $\Sigma \subset \mathbf{R}=(-\infty, \infty)$. (By the support of $w$ we mean the closure of the set where $w$ is positive.) Assume that $w(x)$ vanishes at points of $\Sigma$; that is, $Z:=\{x \in \Sigma: w(x)=0\} \neq \emptyset$ (or, in case $\Sigma$ is unbounded, then $|x| w(x) \rightarrow 0$ as $|x| \rightarrow \infty)$. If $P_{n}$ is an arbitrary polynomial of degree at most $n$, then the sup norm over $\Sigma$ of the weighted polynomial $[w(x)]^{n} P_{n}(x)$ actually "lives" on some compact set $S \subset \Sigma-Z$ which is independent of $n$ and $P_{n}$. The question is to determine the smallest such set $S$.

For example, if $w(x)=x^{\theta /(1-\theta)}$ with $\Sigma=[0,1], 0<\theta<1$, then, as shown in $[2,8], S$ is the subinterval $\left[\theta^{2}, \mathbf{1}\right]$.

In this paper we use potential theoretic methods to show how $S$ can be obtained for a class of weight functions. The assumptions on $w$ are given in

DEFINITION 1.1. Let $w: \mathbf{R} \rightarrow[0,+\infty)$. We say that $w$ is an admissible weight function if each of the following properties holds:

(i) $\Sigma:=\operatorname{supp}(w)$ has positive capacity.

(ii) The restriction of $w$ to $\Sigma$ is continuous on $\Sigma$.

(iii) The set $Z:=\{x \in \Sigma: w(x)=0\}$ has capacity zero.

(iv) If $\Sigma$ is unbounded, then $|x| w(x) \rightarrow 0$ as $|x| \rightarrow \infty, x \in \Sigma$.

Here, and throughout the paper, the term "capacity" means inner logarithmic capacity (cf. $\left[\mathbf{1 0}\right.$, p. 55]). For any set $E \subset \mathbf{R}^{2}$, its capacity will be denoted by $C(E)$. If $K$ is a compact set with positive capacity, then $\nu_{K}$ denotes the unique unit equilibrium measure on $K$ with the property that (cf. $[10$, p. 60])

$$
\int_{K} \log |x-t| d \nu_{K}(t)=\log C(K)
$$

quasi-everywhere (q.e.) on $K$. (A property is said to hold q.e. on a set $A$ if the subset $E$ of $A$ where it does not hold satisfies $C(E)=0$.)

Received by the editors February 6, 1984 and, in revised form, May 23, 1984.

1980 Mathematics Subject Classification. Primary 41A25, 31B15; Secondary 33A65.

${ }^{1}$ Research supported in part by the National Science Foundation.

(C)1984 American Mathematical Society $0273-0979 / 84 \$ 1.00+\$ .25$ per page 
For an admissible weight $w$, we always set

$$
Q(x):=\log (1 / w(x)) .
$$

Finally, if $K \subset \Sigma-Z$ is compact and $C(K)>0$, we define the $F$-functional of $K$ by the formula

$$
F(K):=\log C(K)-\int_{K} Q d \nu_{K}
$$

The theorems of $\S 2$ show that, for a class of weight functions, $S$ is derived by maximizing the $F$-functional. Also, if $\pi_{m}$ denotes the collection of all polynomials of degree at most $m$ and $\|\cdot\|_{A}$ denotes the sup norm over a set $A$, we describe the asymptotic behavior of the errors in the weighted Chebyshev problem

$$
\begin{aligned}
E_{n}(w):=\inf \left\{\left\|[w(x)]^{n}\left\{x^{n}-p_{n-1}(x)\right\}\right\|_{\Sigma}: p_{n-1} \in \pi_{n-1}\right\} \\
n=1,2, \ldots,
\end{aligned}
$$

as well as asymptotic properties (as $n \rightarrow \infty$ ) of the extremal polynomials $T_{n}(x ; w)=x^{n}+\cdots \in \pi_{n}$ which satisfy

$$
E_{n}(w)=\left\|[w(x)]^{n} T_{n}(x ; w)\right\|_{\Sigma}, \quad n=1,2, \ldots
$$

\section{Statements of main results.}

THEOREM 2.1. Let $w$ be an admissible weight function with support $\Sigma$. Then there exists a compact set $S \subset \Sigma-Z$ with $C(S)>0$ that has the following properties.

(a) For every compact set $K \subset \Sigma-Z$ with $C(K)>0$,

$$
F(K) \leq F(S) \text {, }
$$

where $F$ is defined in (1.3).

(b) If equality holds in (2.1), then $S \subset K$.

(c) For any positive integer $n$, if $P_{n} \in \pi_{n}$ and the inequality

$$
\left|[w(x)]^{n} P_{n}(x)\right| \leq M \quad(M=\text { constant })
$$

holds q.e. on $S$, then it holds q.e. on $\Sigma$.

(d) The errors $E_{n}(w)$ defined in (1.4) satisfy

$$
\left[E_{n}(w)\right]^{1 / n} \geq \exp (F(S)), \quad \forall n=1,2, \ldots
$$

Clearly properties (a) and (b) uniquely determine the set $S=S(w)$ of Theorem 2.1. In the special case when $w(x) \equiv 1$ on $\Sigma$ and $\Sigma$ is compact, then $S$ is just the support of the equilibrium measure $\nu_{\Sigma}$ for $\Sigma$.

Of practical importance is the characterization of $S$ given in

THEOREM 2.2. Assume that, in Theorem 2.1, the set $\Sigma-Z$ is the finite union of disjoint nondegenerate intervals and that $Q(x)$ of $(1.2)$ is convex in each of the components of $\Sigma-Z$. Then the following additional properties hold.

(a) The compact set $S$ of Theorem 2.1 is the finite union of nondegenerate disjoint closed intervals, at most one in each component of $\Sigma-Z$. 
(b) Equality holds in (2.1) if and only if $S \subset K$ and $C(K-S)=0$.

(c) For any positive integer $n$, if $P_{n} \in \pi_{n}$, then

$$
\left\|[w(x)]^{n} P_{n}(x)\right\|_{\Sigma}=\left\|[w(x)]^{n} P_{n}(x)\right\|_{s} .
$$

(d) The errors $E_{n}(w)$ of (1.4) satisfy

$$
\lim _{n \rightarrow \infty}\left[E_{n}(w)\right]^{1 / n}=\exp (F(S)) \text {. }
$$

The proof of Theorem 2.1 follows by showing that $S$ is actually the support of a measure which solves an extremal problem for generalized energy integrals, as we now describe. Let $\mathcal{M}(\Sigma)$ denote the collection of all positive unit Borel measures $\mu$ with $\operatorname{supp}(\mu) \subset \Sigma$, and define

$$
I_{w}[\mu]:=\iint[\log |x-t|-Q(x)-Q(t)] d \mu(x) d \mu(t)
$$

for $\mu \in \mathcal{M}(\Sigma)$. Following methods of Frostman (cf. [10]) we obtain

THEOREM 2.3. Let $w$ be an admissible weight function with support $\Sigma$ and let

$$
V_{w}:=\sup \left\{I_{w}[\mu]: \mu \in \mathcal{M}(\Sigma)\right\} .
$$

Then there exists a unique measure $\mu_{w} \in \mathcal{M}(\Sigma)$ such that $I_{w}\left[\mu_{w}\right]=V_{w}$. Moreover, $S_{w}:=\operatorname{supp}\left(\mu_{w}\right)$ satisfies all the properties stated in Theorem 2.1; that is, $S_{w}=S$.

Concerning the limiting distribution of the zeros of the extremal polynomials $T_{n}(x ; w)$ we have

THEOREM 2.4. With the assumptions of Theorem 2.2, let $\left\{x_{k, n}\right\}_{k=1}^{n}$ denote the zeros of the extremal polynomial $T_{n}(x ; w)$ of $(1.5)$, and let $\nu_{n}$ be the associated unit Borel measure defined by

$$
\nu_{n}(B):=(1 / n)\left|\left\{k: x_{k, n} \in B\right\}\right| \text {. }
$$

Then, in the weak star topology,

$$
\lim _{n \rightarrow \infty} \nu_{n}=\mu_{w},
$$

where $\mu_{w}$ is the extremal measure of Theorem 2.3. Furthermore,

$$
\lim _{n \rightarrow \infty}\left|T_{n}(z ; w)\right|^{1 / n}=\exp \left(\int \log |z-t| d \mu_{w}(t)\right)
$$

uniformly on every compact set of the plane disjoint from the convex hull $[\lambda, \tau]$ of $S$.

3. Applications. For Jacobi weights of the form $w(x)=x^{\theta /(1-\theta)}, 0<$ $\theta<1, \Sigma=[0,1]$, or $w(x)=(1-x)^{\lambda_{1}}(1+x)^{\lambda_{2}}, \lambda_{1}, \lambda_{2}>0, \Sigma=[-1,1]$, maximizing the associated $F$-functional leads to the results of $[2,9,3$ and 7$]$ concerning incomplete polynomials.

For a weight $W$ on $\mathbf{R}$ of the form $W(x)=\exp (-q(x))$, where $q(x)$ is even and convex on $\mathbf{R}$ and $q(x) / \ln x \rightarrow \infty$ as $x \rightarrow \infty$, we can also analyze the extremal problems

$$
e_{n}(W):=\inf \left\{\left\|W(x)\left\{x^{n}-p_{n-1}(x)\right\}\right\|_{\mathbf{R}}: p_{n-1} \in \pi_{n-1}\right\}, \quad n=1,2, \ldots,
$$


and the corresponding extremal polynomials $t_{n}(x ; W)=x^{n}+\cdots \in \pi_{n}$ satisfying $e_{n}(W)=\left\|W(x) t_{n}(x ; W)\right\|_{\mathbf{R}}$. After maximizing the appropriate $F$ functional, Theorem 2.2(c) yields

$$
\left\|W(x) P_{n}(x)\right\|_{\mathbf{R}}=\left\|W(x) P_{n}(x)\right\|_{\left[-a_{n}, a_{n}\right]}, \quad \forall P_{n} \in \pi_{n},
$$

where $a=a_{n}$ is a root of the equation

$$
n=\frac{2}{\pi} \int_{0}^{1} \frac{a x q^{\prime}(a x)}{\sqrt{1-x^{2}}} d x .
$$

Letting $w_{n}(x):=\exp \left(-q\left(a_{n} x\right) / n\right)$ with $\Sigma_{n}:=\operatorname{supp}\left(w_{n}\right)=[-1,1]$, it follows from (3.2) that

$$
e_{n}(W)=a_{n}^{n} E_{n}\left(w_{n}\right), \quad t_{n}\left(a_{n} x ; W\right)=a_{n}^{n} T_{n}\left(x ; w_{n}\right) .
$$

If the weights $w_{n}$ converge uniformly to an admissible weight $w$ on $[-1,1]$, it can be shown that the asymptotic behaviors (as $n \rightarrow \infty)$ of $E_{n}\left(w_{n}\right)$ and $T_{n}\left(x ; w_{n}\right)$ are the same as that for $E_{n}(w)$ and $T_{n}(x ; w)$. These facts lead to the results of [5] for $W(x)=\exp \left(-|x|^{\alpha}\right), \alpha \geq 1$, as well as to $L^{\infty}$-analogue of the $L^{2}$-results in $[\mathbf{1}]$ for $W(x)=\exp (-\exp |x|)$.

\title{
REFERENCES
}

1. P. Erdös, On the distribution of the roots of orthogonal polymomials, Proc. Conf. on Constructive Theory of Functions (G. Alexits and S. B. Steckhin, eds.), Adadémiai Kiadó, Budapest, 1972, pp. 145-150.

2. J. H. B. Kemperman and G. G. Lorentz, Bounds for polynomials with applications, Nederl. Akad. Wetensch. Proc. Ser. A 82 (1979), 13-26.

3. M. A. Lachance, E. B. Saff and R. S. Varga, Bounds for incomplete polymomials vanishing at both endpoints of an interval, Constructive Approaches to Mathematical Models (C. V. Coffman and G. J. Fix, eds.), Academic Press, New York, 1979, pp. 421-437.

4. G. G. Lorentz, Approximation by incomplete polymomials (problems and results), Padé and Rational Approximation: Theory and Applications (E. B. Saff and R. S. Varga, eds.), Academic Press, New York, 1977, pp. 289-302.

5. H. N. Mhaskar and E. B. Saff, Extremal problems for polynomials with exponential weights, Trans. Amer. Math. Soc. 285 (1984), 203-234.

6. E. B. Saff, Incomplete and orthogonal polymomials, Approximation Theory IV (C. K. Chui, L. L. Schumaker and J. D. Ward, eds.), Academic Press, New York, 1983, pp. 219-256.

7. E. B. Saff, J. L. Ullman and R. S. Varga, Incomplete polymomials: an electrostatics approach, Approximation Theory III (E. W. Cheney, ed.), Academic Press, New York, 1980, pp. 769 782.

8. E. B. Saff and R. S. Varga, The sharpmess of Lorentz's theorem on incomplete polynomials, Trans. Amer. Math. Soc. 249 (1979), 163-186.

9. __ On incomplete polymomials, Numerische Methoden der Approximationstheorie (L. Collatz, G. Meinardus and H. Werner, eds.), Internat. Schriftenreihe Numer. Math. 42 Birkhäuser Verlag, Basel, 1978, pp. 281-298.

10. M. Tsuji, Potential theory in modern function theory, Maruzen, Tokyo, 1959.

\author{
Department OF MAThematics, CAlifornia State University, LOS \\ ANGELES, CALIForNia 90032
}

Department of Mathematics, UNiVersity of South Florida, TAMPa, FLORIDA, 33620 